\title{
A PERCEPÇÃO DOS NÚCLEOS DE INOVAÇÃO TECNOLÓGICA DO ESTADO DE SÃO PAULO QUANTO AS BARREIRAS À TRANSFERÊNCIA DE TECNOLOGIA UNIVERSIDADE-EMPRESA
}

\author{
Paulo Renato Pakes ${ }^{*}$, Miguel Ángel Aires Borrás ${ }^{2}$, Ana Lúcia Vitale Torkomian ${ }^{3}$, Aline Okumoto Gomes ${ }^{3}$, Brena Bezerra Silva 3 \\ 1 Programa de Pós-Graduação em Engenharia de Produção, Universidade Federal de São Carlos, 13565-905, São Carlos, Brasil. \\ 2 Departamento de Engenharia de Produção, Universidade Federal de São Carlos, 18052-780, Sorocaba, Brasil. \\ 3 Departamento de Engenharia de Produção, Universidade Federal de São Carlos, 13565-905, São Carlos, Brasil.
}

*E-mail: paulopakes@gmail.com

\begin{abstract}
RESUMO
No Brasil, no que tange ao incentivo à relação universidade-empresa, foi promulgada em 2004 a Lei de Inovação (Lei ${ }^{\circ} 10.973$ de 2004) - que dispõe medidas de incentivo à inovação e à pesquisa científica e tecnológica no ambiente produtivo, com vistas à capacitação e ao alcance da autonomia tecnológica e ao desenvolvimento industrial do País. Esta Lei veio a refletir em mudanças na configuração das universidades bem como na relação universidade-empresa. As universidades tiveram de instituir os Núcleos de Inovação Tecnológica (NIT) bem como se adaptar a outros mecanismos de transferência de tecnologia promulgados pela Lei. Destarte, buscou-se elucidar quais as barreiras à transferência de tecnologia, segundo opinião dos NIT. Por meio de um estudo multicasos observou-se que as percepções dos NIT em relação às barreiras à transferência de tecnologia universidade-empresa apresentam naturezas diferentes apesar de apresentarem congruências em alguns pontos e que as barreiras se referem-se a aspectos de ordem jurídica, políticogovernamental, relacional, econômica, de recursos humanos, operacionais, tecnológicas e mercadológicas.
\end{abstract}

Palavras-chave: Relação universidade-empresa; Núcleo de Inovação Tecnológica; Barreiras à transferência de tecnologia.

\section{Introdução}

Inovações de produto e de processo estimulam o crescimento e produtividade não somente das empresas que as desenvolvem, mas também de outras empresas que as adotam e utilizam. Desta forma, em uma perspectiva sistêmica, seus impactos estendem-se ao setor econômico e de lá para a competitividade nacional e internacional e, por fim, à balança comercial [1]. Portanto, a difusão do conhecimento e da tecnologia, denominada transferência tecnológica, é parte central do processo de inovação [2].

A formação de alianças entre universidades e empresas para transferência de tecnologia pauta-se na utilização de redes de conhecimento para adoção de inovação ou melhoria do desempenho inovativo. Ademais, é um exemplo de rede de conhecimento que permite a realização da solução de problemas específicos associados ao processo da inovação, visto que se reduzem os riscos e incertezas, aumenta-se o acesso a conhecimento e informação externa além de proporcionar acesso a outras bases de recursos que facilitam o aprendizado e a inovação [3].

Assim como empresas e governo, as universidades tradicionais formadoras de capital humano e geradoras de novos conhecimentos - sobretudo nos Estados Unidos (EUA) e na Europa, modificam sua missão e participam ativamente das discussões sobre o desenvolvimento de suas regiões, sobre empreendedorismo e inovação [4-5]. O conceito do modelo teórico da hélice tríplice proposto por Henry Etzkowitz e Loet Leydesdorff fundamenta e respalda a relação UniversidadeIndústria-Governo, uma realidade nas universidades americanas e europeias que trabalham alinhadas às demandas da sociedade [6-7].

As "universidades empreendedoras" são hoje fontes de tecnologia comercial, atuam em conjunto com governo e sociedade no desenvolvimento de suas regiões. São estruturas híbridas em torno do conhecimento e da inovação, hospedam escritórios de transferência de tecnologia, incubadoras, parques científicos e tecnológicos, empresas spin-off e empresas juniores [6,8-9].

No Brasil, no que tange ao incentivo à relação universidade-empresa, foi promulgada em 2004 a Lei de Inovação (Lei no 10.973 de 2004) - que dispõe medidas de incentivo à inovação e à pesquisa científica e tecnológica no ambiente produtivo, com vistas à capacitação e ao alcance da autonomia tecnológica e ao desenvolvimento industrial do País. Esta Lei veio a refletir em mudanças na configuração das universidades bem como na relação universidade-empresa. As universidades tiveram de instituir os Núcleos de Inovação Tecnológica (NIT) bem como se adaptar a outros mecanismos de transferência de tecnologia promulgados pela Lei. A Lei $10.973 / 2004$ foi projetada para prover incentivos à pesquisa e 
inovação e cobrir importantes provisões, tais como acordos de cooperação entre Instituições de Ciência e Tecnologia (ICT) e empresas, o compartilhamento de laboratórios, a incubação de pequenas empresas, a provisão de serviços de $\mathrm{P} \& \mathrm{D}$ às indústrias, a criação de NIT responsáveis pela proteção da propriedade intelectual e a participação de pesquisadores em recompensas econômicas de suas inovações e patrocínio de companhias inovadoras [10].

Destarte, este trabalho realizou-se com vistas ao melhor entendimento sobre como as universidades estão conduzindo processos envolvendo adaptação à Lei 10.973/2004, atualizada em 2016 pela Lei 13.243, de 11/01/2016. A nova lei é resultado de um processo de cerca de cinco anos de discussões entre atores do Sistema Nacional de Inovação (SNI) nos âmbitos das Comissões de Ciência e Tecnologia da Câmara e do Senado. Estas discussões tinham como ponto de partida o reconhecimento e a necessidade de alterar pontos na Lei de Inovação e em outras nove leis relacionadas ao tema, de modo a reduzir obstáculos legais e burocráticos e conferir maior flexibilidade às instituições atuantes neste sistema [11].

Neste sentido, buscou-se elucidar quais as barreiras à transferência de tecnologia, segundo opinião dos NIT. Realizouse o trabalho com vistas a munir o Estado de informações inerentes à proteção e transferência de tecnologia das criações desenvolvidas nos limites das universidades bem como elucidar as barreiras à transferência de tecnologia, no intuito de subsidiar os legisladores de informações para o desenho de políticas e auxiliar os governadores no processo de tomada de decisões referentes à temática.

Portanto, diante de tal e considerando-se a institucionalização dos NIT nas universidades públicas do Estado de São Paulo, buscou-se evidenciar por meio de um estudo multicasos quais são as barreiras à transferência de tecnologia universidade-empresa segundo a perspectiva destes.

\section{Referencial Teórico}

2.1 A constituição do Sistema Nacional de Inovação: A Lei da Inovação e seus efeitos sobre a configuração das universidades brasileiras

Em países com relevante papel do setor governamental na promoção do desenvolvimento econômico e social, em particular no tocante a políticas indutivas de colaboração entre os atores do sistema de inovação, incluindo instituições de pesquisa e o setor empresarial, observou-se a regulamentação de medidas de incentivo à criação de um ambiente propício à inovação. No Brasil, a Lei de Inovação Federal de 2004 foi sancionada como resultado de um processo iniciado em 2001 por meio de um anteprojeto exposto na Conferência Nacional de Ciência e Tecnologia organizada pelo Ministério de Ciência e Tecnologia [12].
A utilização do conhecimento gerado nas universidades representa rica fonte de informação e capacitação para o desenvolvimento de novas tecnologias, resultando no fato de que a transferência de tecnologia entre a universidade e a empresa consiste em um caminho alternativo e complementar para o alcance de uma etapa tecnológica superior das empresas. Entre as diversas formas de transferência de tecnologias resultantes da pesquisa acadêmica, destacam-se o licenciamento de patentes e a criação de novas empresas, sendo que tais mecanismos formais de transferência de conhecimentos e transformação em bens disponíveis ao mercado têm sido considerados a mudança mais significativa nas relações universidade-empresa [12].

O Sistema Nacional de Inovação (SNI) é fortalecido e deve ser desenvolvido como uma rede de instituições públicas e privadas que interagem para promover o desenvolvimento científico e tecnológico de um país, incluindo como agentes as universidades, escolas técnicas, institutos de pesquisa, agências governamentais de fomento, indústrias, associações empresariais e agências reguladoras, num esforço de geração, importação, modificação, adaptação e difusão de inovações [13].

Com vistas a maior interação dos elementos do Sistema Nacional de Inovação instituiu-se a Lei 10.973/2004, denominada Lei da Inovação. O ambiente de inovação brasileiro passa por uma série de mudanças desde então.

Cabe aqui destacar que algumas universidades contavam com escritórios de transferência de tecnologia incumbidos de auxiliar seus pesquisadores a gerenciar os seus pedidos de patentes até dez anos antes da promulgação da Lei. Tal situação justificaria a atual diferença de amadurecimento, estrutura e capacitação entre os NIT atualmente [12]. No entanto, com a obrigatoriedade, instaurada pela Lei 10.973, da instalação dos NIT em todas as ICT, não apenas foram formalizadas suas atividades e funções, como a gestão da inovação tecnológica dentro das universidades passou a ser vista como uma área política estratégica para o País.

Entre os temas tratados pela Lei de Inovação, grande peso é dado ao estabelecimento de mecanismos de incentivo à interação ICT-empresa e ao fortalecimento dos agentes intermediadores dessa relação, como as instituições de apoio (na figura das fundações de apoio - Lei no 8.958/1994) e os chamados núcleos de inovação tecnológica (NITs) [11].

A nova lei avança em diversos pontos na promoção de um ambiente regulatório mais seguro e estimulante para a inovação no Brasil. Entre eles, destacam-se: a formalização das ICTs privadas (entidades privadas sem fins lucrativos) como objeto da lei; a ampliação do papel dos NITs, incluindo a possibilidade de que fundações de apoio possam ser NITs de ICTs; a diminuição de alguns dos entraves para a importação de insumos para pesquisa e desenvolvimento (P\&D); a formalização das bolsas de estímulo à atividade inovativa, entre outros [11-12].

Essa possibilidade dará a essas instituições, entre outras vantagens, maior flexibilidade na gestão de seus recursos 
financeiros (dissociados, portanto, dos orçamentos das ICTs), maior celeridade e possiblidade de atração de perfis e contratação de funcionários mais qualificados em relação às atribuições previstas e, como consequência, maior profissionalismo na gestão da política de C,T\&I das ICTs [11].

Ainda, a Lei faculta à ICT prestar a instituições públicas ou privadas serviços compatíveis com os objetivos desta nas atividades voltadas à inovação e à pesquisa científica e tecnológica no ambiente produtivo. Diante de tal contexto, o momento atual é de uma adequação das estruturas acadêmicas que visa ao aperfeiçoamento de sua gestão tecnológica para o aproveitamento dos resultados de pesquisa acadêmica. Essa adequação à Lei se dá através do estabelecimento de regulamentações internas (inerentes à transferência de tecnologia e à instituição de seus NIT) e da implementação de uma infraestrutura capaz de proteger e comercializar tecnologias universitárias valendo-se de ferramentas de gestão e capacitação requeridas para tais atividades [12].

Frente a essas instituições da Lei, os NIT têm a atribuição de gerenciar toda a propriedade intelectual gerada pela ICT que os constitui, englobando a proteção da propriedade intelectual e a transferência de tecnologia e promover a articulação entre a Universidade e as empresas com o intuito de estimular a inovação tecnológica no Brasil.

Conforme visto, a reconfiguração universidade-empresa proporcionada pela Lei implicou na necessidade das universidades se reestruturarem, com vistas a permitir uma maior interação com o setor privado (criação dos NIT para intermediação da relação universidade-empresa) para que seja possível a transferência tecnológica, seja através de licenciamento de patentes ou criação de startups e spin-offs.

Neste novo ambiente de inovação, as universidades desempenham um papel fundamental, incorporando como parte de sua função acadêmica o desenvolvimento econômico, que se adiciona ao ensino e à pesquisa. Como consequência deste novo compromisso, novas funções são assumidas pelas universidades: projetos tecnológicos em cooperação com empresas; comercialização de resultados de pesquisa; patenteamento de produtos e processos; e licenciamento de tecnologias. Portanto, neste contexto, a cooperação universidade-empresa deixa de ser uma atividade informal e ocasional, como ocorria no passado, para adquirir um caráter formal, frequente e planejado, com relações regidas por contratos, que incluem a regulação dos possíveis direitos de propriedade intelectual gerados no âmbito dos projetos cooperativos de pesquisa [12].

Desta forma, diante da relevância socioeconômica que adquire a proteção da propriedade intelectual, torna-se fundamental que as ICT tenham consciência sobre a necessidade de proteger os resultados da pesquisa antes de publicá-la, para evitar a perda dos direitos e, consequentemente, que os possíveis dividendos derivados da invenção venham a ser apropriados por terceiros, resultando no prejuízo econômico devido a não exploração dos inventos [14]. Este tipo de pressão remete ao sujeito que atua no setor de transferência de tecnologia em núcleos de inovação tecnológica - geralmente denominados agentes de inovação, agentes de transferência ou agentes de parceria.

Na prática, os NIT têm a atribuição de gerenciar toda a propriedade intelectual gerada pela ICT que os constitui, englobando a proteção da propriedade intelectual e a transferência de tecnologia e promover a articulação entre a Universidade e as empresas com o intuito de estimular a inovação tecnológica no Brasil.

\subsection{Perspectiva dos envolvidos no processo de transferência de tecnologia universidade-empresa}

Enquanto atores da ciência, os acadêmicos e universitários de uma maneira geral - quando envolvidos no relacionamento com outros atores no sistema nacional de inovação - estão tipicamente interessados em coisas como investimentos para instituições de pesquisa e projetos, o desenvolvimento de conhecimento científico e carreiras (publicações, liderança profissional), o crescimento de disciplinas ou áreas temáticas e o treinamento de jovens pesquisadores [15].

Um aspecto significante do processo de transferência de tecnologia envolve a consideração das ações, motivos e perspectivas dos cientistas/grupos de pesquisa, administradores universitários e empresa/empreendedores [16], conforme representado na tabela 1 .

Portanto, a motivação primária dos cientistas universitários se traduz no reconhecimento junto à comunidade acadêmica, que geralmente emana de publicações em revistas de relevância, apresentações em conferências de prestígio e recompensas financeiras. Os membros do corpo docente podem também ser motivados por ganhos financeiros pessoais e/ou desejo de obter financiamento adicional para estudantes de pósgraduação ou equipamentos para laboratório [16].

A motivação primária do NIT e da administração universitária é de resguardar a propriedade intelectual da universidade, enquanto ao mesmo tempo é de comercializar a propriedade intelectual para empresas/empreendedores. As motivações secundárias incluem assegurar fundos adicionais de pesquisa para a universidade através de royalties e taxas de licenciamento, acordos de patrocínio de pesquisa e um íntrinseco desejo de promover a difusão tecnológica (o objetivo de leis de inovação como o Bay-Dole Act, dos EUA, e a Lei 10.973/2004, no Brasil). Os gerentes do NIT, assim como outros administradores universitários, trabalham dentro de uma plataforma de trabalho burocrática dentro das universidades. 
Tabela 1 - Ações, motivações e perspectiva dos stakeholders envolvidos no processo de transferência de tecnologia universidade-empresa [16].

\begin{tabular}{|c|c|c|c|c|}
\hline Stakeholder & Ações & Motivações primárias & Motivações secundárias & Perspectiva \\
\hline $\begin{array}{l}\text { Cientista } \\
\text { Universitário }\end{array}$ & $\begin{array}{l}\text { Descoberta de novos } \\
\text { conhecimentos }\end{array}$ & $\begin{array}{l}\text { Reconhecimento junto à } \\
\text { comunidade científica - } \\
\text { publicações, recompensas. }\end{array}$ & $\begin{array}{l}\text { Ganho financeiro e desejo de } \\
\text { investimentos adicionais em pesquisa } \\
\text { (principalmente para estudante de pós- } \\
\text { graduação e materiais laboratórios) }\end{array}$ & Científica \\
\hline NIT & $\begin{array}{l}\text { Trabalhar com } \\
\text { membros da } \\
\text { universidade } \\
\text { empresas/empreendedo } \\
\text { res para estruturar } \\
\text { contratos/negócios }\end{array}$ & $\begin{array}{l}\text { Proteger e comercializar } \\
\text { propriedade intelectual da } \\
\text { universidade }\end{array}$ & $\begin{array}{l}\text { Facilitar difusão tecnológica e } \\
\text { assegurar fundos adicionais à pesquisa }\end{array}$ & Burocrática \\
\hline $\begin{array}{l}\text { Empresa/ } \\
\text { Empreendedor }\end{array}$ & $\begin{array}{l}\text { Comercializar novas } \\
\text { tecnologias }\end{array}$ & Ganho financeiro & $\begin{array}{l}\text { Manter controle sobre propriedade de } \\
\text { tecnologias }\end{array}$ & $\begin{array}{l}\text { Orgânica/ } \\
\text { Empreendedora }\end{array}$ \\
\hline
\end{tabular}

No entanto, os interesses dos clientes externos (empresas/empreendedores) são diferentes dos outros envolvidos no processo de transferência de tecnologia (NIT/cientistas/grupos de pesquisa) [16].

Já as empresas e empreendedores procuram comercializar tecnologias desenvolvidas em universidades com vistas a ganhos financeiros. Para tal, estes almejam a exclusividade dos direitos de explorar a produção de tais tecnologias. As empresas e os empreendedores também expressam grande preocupação em relação ao tempo de mercado (time to market) uma vez que os benefícios finais do produto e do processo de inovação dependem da comercialização do produto ou do aperfeiçoamento do novo processo de produção antes que os competidores o façam. Além disso, as empresas/empreendedores estão também preocupados quanto à manutenção do controle da propriedade sobre as tecnologias licenciadas [16].

As diferenças nas ações, motivações e perspectivas destes três elementos-chave no processo de transferência de tecnologia universidade-empresa sublinham a potencial importância dos fatores organizacionais no que tange à eficácia do gerenciamento universitário da propriedade intelectual.

Para superar as barreiras críticas à efetiva transferência de tecnologia, tornam-se necessárias as seguintes ações [16]:

a) as universidades/NIT devem melhorar seu entendimento das necessidades de seus verdadeiros "consumidores", ou seja, das empresas que podem comercializar suas tecnologias de forma potencial;

b) as universidades/NIT devem adotar posturas mais flexíveis ao negociar acordos de transferência tecnológica e simplificar suas políticas e procedimentos de transferência de tecnologia universidade-empresa;

c) as universidades/NIT devem contratar gestores/administradores de pesquisa com visão estratégica, que possam servir como "atravessadores de fronteiras";

d) as universidades devem dedicar recursos adicionais ao NIT e ao patenteamento; e) as universidades/NIT devem aumentar as recompensas para participação de membros do corpo docente no processo de transferência de tecnologia universidade-empresa através da valorização de patentes e licenças em promoção e decisão de posse. Além disso, devem permitir aos membros do corpo docente manter uma parcela maior da receita de licenciamento (em oposição ao seu departamento ou universidade);

f) as universidades/NIT devem reconhecer o valor dos relacionamentos pessoais e networks sociais, envolvendo cientistas, estudantes de pós graduação e alunos.

Tendo em vista que a perspectiva dos envolvidos no processo de transferência de tecnologia universidade empresa são diferentes [16] e levando em consideração que os NIT do País ainda se encontram em estruturação $[12,17]$ este trabalho buscou investigar quais as dificuldades encontradas por estes no processo de transferência de tecnologia.

\section{Metodologia}

Os métodos de pesquisa devem ser pensados como uma estrutura e orientação geral para condução de uma investigação [18]. Para esta pesquisa o método escolhido em função de sua adequação com a abordagem e contingências da pesquisa foi o estudo de caso.

Os estudos de caso se classificam em três possibilidades: (1) exploratório, (2) descritivo, e (3) explanatório (ou explicativo). No primeiro caso, deseja-se conhecer com maior profundidade questões pouco conhecidas. Já o segundo, está mais preocupado com a descrição da situação. E o terceiro caso se volta a possibilidades de explicação de causas [19].

No entanto, tais definições não carregam nenhum peso hierárquico, e estas estratégias muitas vezes se sobrepõem, não havendo, portanto, uma limitação objetiva entre elas. Cada estratégia, por sua vez, tende a resolver problemas diferentes [19]. 
Esta pesquisa adotou a abordagem descritiva e explanatória, tendo em vista que se buscou descrever a situação. Dessa forma, este estudo pode ser classificado como estudo de caso múltiplo descritivo.

Uma vez classificada a pesquisa, este estudo foi conduzido em três grandes fases, conforme Figura 1.

Uma vez que não há uma ampla gama de estudos preliminares com o escopo estrito deste trabalho sobre o tema da pesquisa, o estudo possui caráter exploratório, além de características de um estudo descritivo. Foram seguidos os pressupostos do método qualitativo objetivando compreender fatos em profundidade. O delineamento da pesquisa é de múltiplos casos com foco no entendimento da dinâmica presente de fatos recentes [20] e em fenômenos contemporâneos inseridos em um contexto da vida real em que o pesquisador não tem domínio das variáveis [19].

Iniciou-se a abordagem do tema por meio de um levantamento bibliográfico sobre transferência de tecnologia, sistema nacional de inovação bem como sobre a legislação relacionada aos temas. Na sequência elaborou-se um instrumento de pesquisa que permitiu a caracterização das barreiras à transferência de tecnologia universidade-empresa. Foi realizado teste-piloto seguido de um websurvey de perguntas abertas aos NIT de quatro universidades públicas localizadas no Estado de São Paulo. O período de aplicação da websurvey compreendeu os meses de março/2016 a agosto/2016.

A compilação dos dados permitiu obter um panorama sobre a percepção desses NIT sobre as barreiras à transferência de tecnologia universidade-empresa. Os resultados do trabalho podem auxiliar a tomada de decisões e/ou elaboração de políticas tangentes à transferência de tecnologia universidade-empresa.

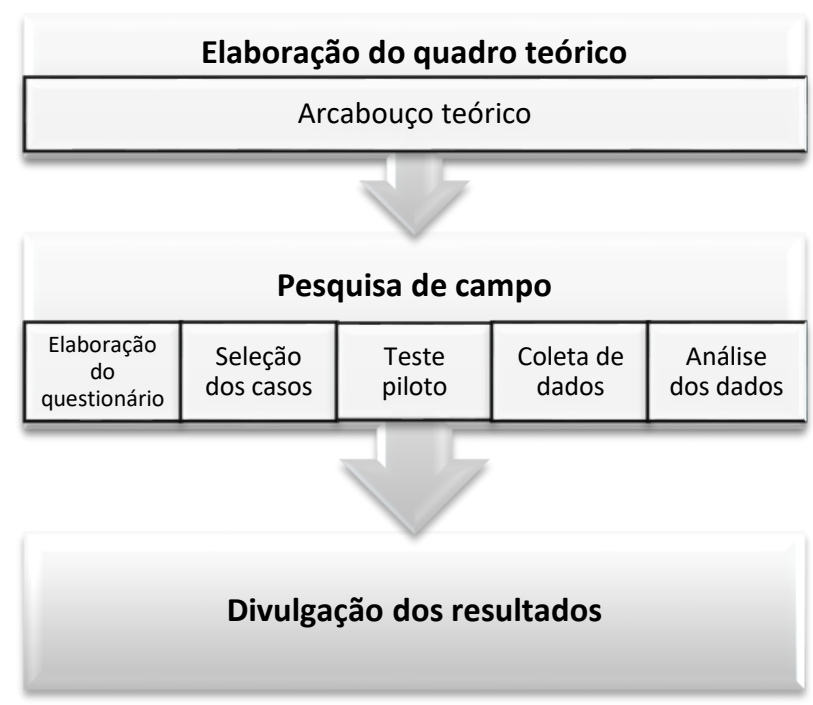

Figura 1 - Delineamento da pesquisa.

\section{Resultados e discussões}

Nas subseções seguintes apresentam-se as dificuldades no processo de transferência de tecnologia universidade-empresa segundo cada um dos NIT das universidades participantes da pesquisa.

\subsection{NIT A}

Segundo o NIT A, as principais dificuldades no processo de transferência de tecnologia universidade-empresa são as seguintes:

a) descontinuidades na atuação de sucessivos governos na implementação consistente de uma política nacional de ciência, tecnologia e inovação;

b) falta de compreensão do papel da propriedade intelectual como ferramenta de difusão da tecnologia científica à tecnologia empresarial;

c) falta de políticas internas na instituição que promovam as ações de comercialização e transferência de tecnologia;

d) necessidade de contratação e capacitação de pessoal qualificado de forma efetiva e permanente;

e) necessidade de profissionais com competências e habilidades para transferência e negociação das tecnologias protegidas, tanto no ambiente empresarial quanto universitário;

f) problemas relativos à sustentabilidade financeira do NIT e recursos escassos para ações internas; e a

g) necessidade de encontrar as pessoas certas, com perfis apropriados, tanto no ambiente empresarial quanto universitário, a fim de promover o desenvolvimento de novas tecnologias visando sua efetiva comercialização para a sociedade.

\subsection{NIT B}

Segundo o NIT B, as principais dificuldades no processo de transferência de tecnologia universidade-empresa são as seguintes:

h) determinadas tecnologias, por mais potencial que tenham, não possuem grupo de pesquisadores em atividades para a sua devida continuidade; quando há interesse da empresa, não há suporte necessário para a otimização da tecnologia ou adequação;

i) tempo de análise e validação da interação, por parte da universidade como por parte da empresa. Os processos de validação de parceria podem ser demorados, considerandose as etapas e normas internas; alguns processos, por sua natureza, apresentam uma maior facilidade de análise (prestação de serviços, etc.), enquanto outros apresentam uma maior complexidade, em especial se envolverem mais de duas instituições;

j) considerando-se tecnologias altamente inovadoras, o processo de valoração das mesmas, para uma oferta tecnológica poderá ser um pouco mais extenso, uma vez que 
não há similar no mercado, o que acabará gerando uma nova oportunidade e diferencial econômico, porém demandará maiores pesquisas e trabalhos sobre a tecnologia, para a sua efetiva e justa comercialização;

k) muitas tecnologias estão em estágio embrionário de desenvolvimento, mesmo considerando todo seu potencial inovador, o que acarretará em maiores dispêndios por parte de um licenciante para se averiguar a sua viabilidade, ou seja, realizar a prova de conceito da tecnologia, em condições que atendam às demandas específicas e legais, o que pode levar tempo e consumir recursos;

1) com relação ao último item, são necessários maiores aportes públicos em inovação, viabilizando recursos e linhas de fomento aos novos potenciais desenvolvimentos, para que descobertas e tecnologias potenciais possam ser ofertadas ao mercado, trazendo benefícios, desenvolvimento econômico e diferencial tecnológico.

\subsection{NIT C}

Segundo o NIT C, as principais dificuldades no processo de transferência de tecnologia universidade-empresa se referem a:

m) cenário embrionário de inovação por parte das empresas brasileiras;

n) aspectos técnicos podem causar dificuldades na viabilização da transferência de tecnologia, como a patente ser indeferida, ter um alto custo de produção ou problemas no desenvolvimento;

o) podem ocorrer aspectos mercadológicos de impedimento, tais como dificuldade na inserção da tecnologia e elevada barreira de entrada; tecnologia pode ter ficado ultrapassada e, por isso, não é atrativa do ponto de vista mercadológico; poderão ocorrer mudanças mercadológicas que impeçam a transferência;

p) as dificuldades estão no não cumprimento das obrigações, bem como nas dificuldades financeiras de pequenas empresas, na falta de interesse e na utilização da tecnologia mediante o pagamento de royalties; outra dificuldade é uma possível mudança de estratégia comercial e principalmente a falta de investimentos necessários para que a transferência se concretize;

q) falta de infraestrutura da empresa para inovação, bem como a incapacidade de absorver a tecnologia e a dificuldade em transformar a tecnologia em um produto comercial também são dificuldades comuns no desenvolvimento do processo como um todo;

r) mesmo após um contato prévio e o alinhamento de informações, pode haver dificuldades de alinhamento entre as expectativas da empresa e da Universidade, o que dificulta $\mathrm{o}$ andamento do processo.

s) baixo comprometimento por parte do pesquisador e a falta de suporte esperado também são fatores negativos para o processo de transferência de tecnologia. t) pela agência de inovação pode haver a dificuldade estratégica, em que pode haver uma falta de definição sobre o objetivo da licença - como investimentos e desenvolvimento; as dificuldades operacionais em se realizar uma transferência de tecnologia consistem na falta de informação suficiente de mercado; dificuldade no cumprimento de prazos estabelecidos; troca constante de negociador, por vezes bolsistas; falta de check list do plano de trabalho; e falta de acompanhamento pós-venda;

u) questões de negociação e contratuais podem também representar dificuldades para a efetiva transferência de tecnologia, por exemplo: dificuldade em acertar os royalties ou o estabelecimento de royalties incompatíveis com a realidade da transação;

v) carência de recursos humanos capacitados para realizar transferência de tecnologia e as limitações de orçamento dos NIT para contratar e manter profissionais com essa qualificação.

\subsection{NIT D}

Segundo o NIT D, as principais dificuldades no processo de transferência de tecnologia universidade-empresa se referem a:

a) falta de interlocução do lado empresarial que não está habituado e em sua maioria não tem mão de obra qualificada para tratar sobre o tema;

b) o ambiente jurídico não é favorável, sendo um grande obstáculo;

c) empresas desistem das negociações após verificar que terão de arcar com custos dessas pesquisas (a ideia de custo financeiro em curto prazo e de retorno em longo prazo reduz o investimento em pesquisa);

d) infraestrutura deficiente quanto às informações sobre patentes e sua transposição ao mercado;

e) maior incorporação dos pesquisadores e professores na configuração da exploração comercial;

f) falta de orientação das atividades de pesquisa às necessidades do mercado;

g) falta de cooperação com empresas em áreas de P\&D intensivas;

h) otimização da comercialização, por meio de sistemas flexíveis de estímulos e das redes de contato.

\section{Conclusões}

Este trabalho teve como objetivo analisar as dificuldades na transferência de tecnologia universidade-empresa sob a ótica dos Núcleos de Inovação Tecnológica. A partir da análise exploratória constatou-se que:

a) as percepções dos em relação às barreiras à transferência de tecnologia universidade-empresa apresentam naturezas diferentes apesar de apresentarem congruências em alguns pontos; 
b) as barreiras à transferência de tecnologia pontuadas pelos NIT se referem-se a aspectos de ordem jurídica, políticogovernamental, relacional, econômica, de recursos humanos, operacionais, tecnológicas e mercadológicas;

c) junto ao empresariado há requisições inerentes a maior interlocução e ao maior cumprimento de obrigações contratuais;

d) junto ao governo há requisições inerentes a maior continuidade/solidez de políticas de $\mathrm{C}, \mathrm{T} \& \mathrm{I}$, a maior percepção sobre a importância da propriedade intelectual como ferramenta de difusão tecnológica, bem como maior aporte de recursos públicos em inovação;

e) junto à comunidade acadêmica há requisições inerentes aà maior percepção da propriedade intelectual como ferramenta de difusão tecnológica, qualificação de profissionais para transferência de tecnologia, maior participação dos pesquisadores e professores na configuração da exploração comercial e maior orientação das atividades de pesquisa às necessidades do mercado;

f) quanto às dificuldades operacionais, estas são inerentes à ausência de pessoal qualificado para atuar em transferência de tecnologia, baixa sustentabilidade financeira do NIT, falta de definição de objetivo sobre objetivo da licença; falta de informação suficiente sobre o mercado; não cumprimento de prazos estabelecidos; troca constante de negociador, por vezes bolsistas; falta de check-list do plano de trabalho; dificuldades no processo de valoração de tecnologias; falta de conhecimento do pós-venda; dificuldades no estabelecimento de royalties; necessidade de maior incorporação dos pesquisadores e professores na configuração da exploração comercial

g) quanto às barreiras mercadológicas, estas são inerentes ao cenário empresarial de inovação, ainda em estágio embrionário; mudanças no ambiente; aspectos técnicos inerentes à inovação (dificuldade de aplicação da tecnologia, tecnologia ultrapassada); falta de interesse em tecnologias mediante o pagamento de royalties; não cumprimento de obrigações contratuais; possível mudança na estratégia comercial da empresa; falta de infraestrutura da empresa para inovação; incapacidade da empresa de absorver tecnologia; dificuldades no alinhamento das expectativas da empresa e da universidade; tecnologias em estágio embrionário de desenvolvimento; empresas desistirem de negociações no meio do processo; desalinhamento das pesquisas universitárias junto às necessidades do mercado e falta de cooperação com empresas de P\&D intensivo.

\section{Agradecimentos}

Agradecimentos à Associação de Universidades Grupo Montevidéu, à Semana de Engenharia de Produção SulAmericana e à Universidade de Santa Cruz do Sul pela oportunidade de publicação e premiação deste artigo científico, e à CAPES pelo apoio financeiro.

\section{THE HURDLES OF THE TECHNOLOGY \\ TRANSFER OFFICES FROM SÃO PAULO STATE IN RELATION TO UNIVERSITY-INDUSTRY TECHNOLOGY TRANSFER}

ABSTRACT: In Brazil, the Innovation Law (Law 10.973 of 2004) was promulgated in 2004 with regard to the incentive to the university-company relationship - which provides measures to encourage innovation and scientific and technological research in the productive environment, with objective of training and the reaching technological autonomy and country's industrial development. This law made changes in the configuration of universities as well as in the university-company relationship. Universities had to establish Technological Innovation Centers (NITs) as well as adapt to other technology transfer mechanisms promulgated by the Law. The aim of this paper was to elucidate the hurdles to technology transfer, according to NIT opinion. Through a multi-level study, it was observed that NIT perceptions regarding the barriers to university-company technology transfer are different in nature although they present congruences in some points and that the barriers refer to legal, political, Governmental, relational, economic, human, operational, technological and marketing resources.

Keywords: University-Industry Relations; Technology Transfer Office; Technology Transfer Hurdles.

\section{Referências}

[1] HAGE, J.; MEEUS, M. T. H. Innovation, Science, and Institutional Change: A research handbook. Orgs.: Hage, J.; Meeus, M., Oxford University Press, 2006. Jones, O.; Tang, N. Innovation in product and process: the implications for technology strategy. International Journal of Manufacturing Technology and Management, v. 1, s/n, p. 464-77, 2000.

[2] OCDE, Organização para a Cooperação e Desenvolvimento Econômico. Manual de Oslo. $3^{\mathrm{a}}$ ed. FINEP/OECD, 2005.

[3] MEEUS, M. T. H.; FABER, J. Interorganizational Relations and Innovation: A review and a theoretical extension. In: Innovation, Science, and Institutional Change: A research handbook. Orgs.: Hage, J.; Meeus, M., Oxford University Press, 2006.

[4] GOLDSTEIN, H.; DRUCKER, J. The Economic Development Impacts of Universities on Regions: Do Size and Distance Matter? Economic Development Quarterly, v. 20, n. 22, 2006.

[5] ETZKOWITZ, H. Hélice Tríplice: Universidade-Indústria-Governo - Inovação em Movimento, 1a ed., Porto Alegre: EDIPUCRS, 2009. 
[6] LEYDESDORFF, L.; ETZKOWITZ, H. Emergence of a Triple Helix of university - industry - government relations. Science and Public Policy, v. 23, n. 5, p. 279-286, 1996

[7] ETZKOWITZ, H.; LEYDESDORFF, L. The dynamics of innovation: from national systems and "mode 2" to a triple helix of university-industry-government relations. Research Policy, v. 29, s/n, 2000.

[8]GOLDSTEIN, H.; DRUCKER, J..Assessing the Regional Economic Development Impacts of Universities: A Review of Current Approaches, International Regional Science Review, v. 30, n. 20, 2007.

[9] WARREN, A.; HANKE, R.; TROTZER, D. Models for university technology transfer: resolving conflicts between mission and methods and the dependency on geographic location. Cambridge Journal of Regions, Economy and Society, v. 1, p. 219-232, 2008.

[10] MACULAN, A. M.; MELLO, J. M. C. University start-ups for breaking lockins of the Brazilian Economy. Science and Public Policy, v. 36, n. 2, p. 109-14, 2009.

[11] RAUEN, C. V. O novo marco legal da inovação no Brasil: o que muda na relação ICT-empresa. Disponível em <https://www.almg.gov.br/export/sites/default/acompanhe/eventos/hotsites/2016/f orum_tecnico_startups/documentos/material_de_referencia/02artigo_novo_marco _legal_inovacao_cristiane_rauen.pdf>. Acesso em 3 de março de 2016.

[12] GARNICA, L. A.; TORKOMIAN, A. L. V. Gestão de tecnologia em universidades: uma análise do patenteamento e dos fatores de dificuldade e de apoio à transferência de tecnologia no Estado de São Paulo. Gestão e Produção, São Carlos, v. 16, n. 4, p. 624-638, 2009.

[13] NELSON, R. National Innovation Systems: a comparative analysis. Nova York, Oxford, Oxford University, 1993.

[14] RITTER DOS SANTOS, M. E. La Gestión de la Transferencia de Tecnología de la Universidad al Sector Productivo: um modelo para Brasil. Tese (Doutorado em Ciências da Administração) - Programa de Posgrado en Ciencias de la Administración, Universidad Nacional Autónoma de México, México, 2005.

[15] KUHLMANN, S.; SHAPIRA, P. How is innovation influenced by science and technology policy governance? Transatlantic comparisons. In: Innovation, Science, and Institutional Change: A research handbook. Orgs.: Hage, J.; Meeus, M., Oxford University Press, 2006.

[16] SIEGEL, D.; WALDMAN, D.; LINK, A. Assessing the impact of organizational practices on the relative productivity of university technology transfer offices: an exploratory study. Research Policy, v. 32, n. 1, p. 27-48, 2003.

[17] RITTER DOS SANTOS, M. E.; TORKOMIAN, A. L. V. Technology transfer and innovation: The role of the Brazilian TTO s. International Journal of Technology Management \& Sustainable Development, v. 12, p. 89-111, 2013.

[18] BRYMAN, A. Research methods and organization studies. London: Unwin Hyman, London, 1989. 283 p.

[19] YIN, R. K. Estudo de Caso: planejamento e métodos. 4. ed. Porto Alegre: Bookman, 2010. 248 p.

[20] EISENHARDT, K. M. Building theories from case study research. Academy of Management Review. v. 14, n. 4, p. 532-550, 1989. 